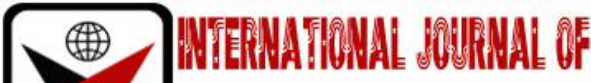

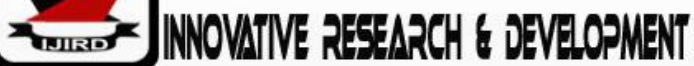

ISSN 2278 - 0211 (Online)

\section{Strength of Existing Buildling against 2017 Indonesian Earthquake Sources and Earthquake Hazards Map}

\section{Tranggono}

Lecturer Department of Civil Enginering, Yos Soedarso University, Surabaya, Indonesia

\begin{abstract}
:
In connection with the issuance of the 2017 Indonesian Earthquake and Hazard Sources Map, trying to recalculate the strength of the Existing Building due to changes in the 2017 Indonesian Earthquake Sources and Hazard Map is needed. Considering the physical work done only partially, it is necessary to review the design. From the results of the analysis it is necessary to improve the structure of the building including; 60/ 60 reinforcement column, reinforcement needs to be added, reinforcing beams lengthening 30/50, reinforcement need to be added, shear wall dimensions and reinforcement need to be added, some foundations on the shear wall need to be increased, while 35/ 70 dimensional beams and reinforcement still meet the requirements.
\end{abstract}

Keywords: Map of 2017 Indonesian earthquake source and hazard, reinforcement, shear wall

\section{Introduction}

\subsection{Background}

With the change in the 2010 Earthquake Map into the 2017 Indonesian Earthquake Source and Earthquake Hazard Map, there will be changes in earthquake parameters that affect the strength of the building that has been designed. Considering the above it is necessary to re-evaluate the buildings that have been designed with the 2010 Earthquake Map.

1.2. Aim

Recalculate the strength of existing buildings in the style of the 2017 Earthquake source and Hazard Map.

1.3. Benefits

To find out the strength of the existing building againts the 2017 Indonesian Earthquake Source and Hazard Map,

\subsection{Restricting the Problem}

Location of building is in Surabaya with soft soil conditions. Number of floors are 6 and the building functions is for hospitals. Building area are $14 \times 80 \mathrm{~m}^{2}$. The dimensions of the main column are $60 \times 60 \mathrm{~cm}^{2}$, transverse beam $35 / 70 \mathrm{~cm} 2$, beam length $30 / 50 \mathrm{~cm}^{2}$, thickness of shear wall $35 \mathrm{~cm}$ and $25 \mathrm{~cm}$, foundation borepile spun $\Phi 50 \mathrm{~cm}$. The 1st floor height is $6 \mathrm{~m}$, while the floor above is typically $4 \mathrm{~m}$. With the number of reinforcement and dimensions as follows :

- Reinforcement installed in 60x60 $\mathrm{cm}^{2}$ columns on the 1st to 6th floors is; $16 \mathrm{D} 22$, Ace $=6064 \mathrm{~mm}^{2}$, stirrup $2 \Phi$ $10-100$.

- Reinforcement installed in $35 \times 70 \mathrm{~cm}^{2}$ transverse beam, tensile reinforcement $6 \mathrm{D} \mathrm{22}$, Ace $=2280 \mathrm{~mm}^{2}$, compressive reinforcement 3 D 22, Ace $=1140$ mm2, stirrup $\Phi 10$ - 100 and $10-120$.

- Reinforced beams are extended 30x50 $\mathrm{cm}^{2}$, tensile reinforcement $4 \mathrm{D} \mathrm{19}$, Ace $=1132 \mathrm{~mm}^{2}$, compressive reinforcement 2 D 19, As =566 $\mathrm{mm}^{2}$, stirrups $\Phi 10$ - 100 and $10-120$.

- Reinforcement is installed on the shear wall $35 \mathrm{~cm}$, vertical reinforcement 2 D 16, distance $150 \mathrm{~mm}$, horizontal reinforcement $2 \Phi 12$, distance $150 \mathrm{~mm}$.

- Reinforcement is installed on a shear wall of $25 \mathrm{~cm}$, vertical reinforcement is 2 D 16, distance is $200 \mathrm{~mm}$, horizontal reinforcement is $2 \Phi 12$, distance is $150 \mathrm{~mm}$.

- Borepile foundation $\Phi 50 \mathrm{~cm}$, depth of MTA $34 \mathrm{~m}$

\section{Literature Review}

\subsection{Earthquake Analysis Stages}

- Determining Building Risk Categories 
- Determine the priority factor

- Determining earthquake response spectral parameters for short periods $\left(\mathrm{S}_{\mathrm{s}}\right)$

- Determine earthquake spectra response parameters for a 1 second period $\left(\mathrm{S}_{1}\right)$

- Determining Land Site Classification (SA - SF)

- Determining Land Site Coefficient Factors $\left(\mathrm{F}_{\mathrm{a}}, \mathrm{F}_{\mathrm{V}}\right)$

- Calculates the spectral acceleration parameters for short period design ( $\left.\mathrm{S}_{\mathrm{DS}}\right)$

- Calculates the spectral acceleration parameters for a 1 second period design ( $\left.\mathrm{S}_{\mathrm{D} 1}\right)$

- $\quad$ Select system and structure parameters $\left(\mathrm{R}_{1} \mathrm{C}_{d}, \Omega_{0}\right)$

- $\quad$ System structure evaluation related to configuration irregularities

- Determine diaphragm flexibility (flexible, semi-rigid, rigid)

- Determine the redundancy factor $(\rho)$

- Determine lateral style analysis procedures

- Calculate lateral loads

2.2. Equivalent Static Load Analysis

Basic Seismic Load $\rightarrow \mathrm{V}=\mathrm{C}_{\mathrm{s}} \mathrm{W}$

Equivalent static earthquake force distribution :

$\mathrm{F}_{\mathrm{X}}=\mathrm{C}_{\mathrm{VX}} \mathrm{V}$

$\mathrm{C}_{\mathrm{VX}}=\frac{\mathrm{Wxhx^{ \textrm {k } }}}{\sum_{\mathrm{i}}^{\mathrm{n}} \mathrm{Wi} \mathrm{hi}^{\mathrm{k}}}$

$\mathrm{F}=$ Earthquake Load

$\mathrm{C}_{\mathrm{V}}=$ Distribution coefficient

$\mathrm{h}_{\mathrm{i}}=$ High floor on level $\mathrm{i}$

$\mathrm{W}_{\mathrm{i}}=$ Effective weight on the floor $\mathrm{i}$

$\mathrm{K}$ is for calculate static equivalents

2.3. Spectra Response Analysis

Determine Vibration Time(T)

$\mathrm{T}_{\mathrm{S}}=\mathrm{S}_{\mathrm{D} 1} / \mathrm{S}_{\mathrm{DS}}$

$\mathrm{T}_{0}=0,2\left(\mathrm{~S}_{\mathrm{D} 1} / \mathrm{S}_{\mathrm{DS}}\right)$

Determining Response Spectra of Design Acceleration $\left(\mathrm{S}_{\mathrm{a}}\right)$

For $\quad \mathrm{T}<\mathrm{T}_{0} \rightarrow \mathrm{Sa}=\mathrm{S}_{\mathrm{DS}} \mathrm{x}\left[0,4+0,6 \frac{\mathrm{T}}{\mathrm{To}}\right]$

For $\quad \mathrm{T}<\mathrm{T}_{0}<\mathrm{T}_{\mathrm{S}} \rightarrow \mathrm{Sa}=\mathrm{S}_{\mathrm{DS}}$

For $\mathrm{T}>\mathrm{T}_{\mathrm{S}} \rightarrow \mathrm{Sa}=\frac{\mathrm{SD} 1}{\mathrm{~T}}$

2.4. Load Combination

Ultimit Load Combination

- $1,4 \mathrm{DL}$

- $1,2 \mathrm{DL}+1,6 \mathrm{LL}+0,5(\mathrm{~A}$ or $\mathrm{R})$

- $1,2 \mathrm{DL}+1,0 \mathrm{LL} \pm 1,6 \mathrm{~W}+0,5(\mathrm{~A}$ or $\mathrm{R})$

- $1,2 \mathrm{DL}+1,0 \mathrm{LL}+1,0 \mathrm{E}$

- $0,9 \mathrm{DL} \pm 1,6 \mathrm{~W}$

- $0,9 \mathrm{DL}+1,0 \mathrm{E}$

Allowed Load Combination

- 1,0DL

- $1,0 \mathrm{DL}+1,0 \mathrm{LL}$

- $1,0 \mathrm{DL}+1,0(\mathrm{~A}$ or $\mathrm{R})$

- $1,0 \mathrm{DL}+0,75 \mathrm{LL}+0,75(\mathrm{~A}$ or $\mathrm{R})$

- $1,0 \mathrm{DL} \pm(0,6 \mathrm{~W}$ or $0,7 \mathrm{E})$

- $1,0 \mathrm{DL} \pm 0,75(0,6 \mathrm{~W}$ or $0,75 \mathrm{E})+0,75 \mathrm{LL}+0,75(\mathrm{~A}$ or $\mathrm{R})$

- $0,6 \mathrm{DL} \pm 0,6 \mathrm{~W}$

- $0,6 \mathrm{DL} \pm 0,7 \mathrm{E}$

2.5. Control of Strength and Stability

- $\quad$ Story Drift and Structural Deformation

- Dimensions and Reinforcement of Columns, Beams

- Shear Wall Dimensions and Reinforcement

- Foundation 


\section{Result and Discussion}

\subsection{Analysis of Earthquake Force Factors}

Risk category: IV ( Hospital )

Spektra respons ;

Locationof Surabaya: $\mathrm{S}_{\mathrm{S}}=0,90$ (Figure D11, Earthquake Map 2017)

$$
\begin{aligned}
& : \mathrm{S}_{1}=0,30 \text { (Figure D12, Earthquake Map 2017) } \\
& : \mathrm{S}_{\mathrm{S}}=0,90 \rightarrow \mathrm{F}_{\mathrm{a}}=0,93 \text { (Table 4, Interpolation, soft soil) } \\
& : \mathrm{S}_{1}=0,30 \rightarrow \mathrm{F}_{\mathrm{V}}=2,80 \text { (Table 5, Interpolation, Soft soil) } \\
& \quad: \mathrm{S}_{\mathrm{DS}}=\frac{2}{3} \mathrm{~F}_{\mathrm{a}} \mathrm{S}_{\mathrm{S}}=\frac{2}{3} \times 0,93 \times 0,90=0,54 \\
& \quad: \mathrm{S}_{\mathrm{D} 1}=\frac{2}{3} \mathrm{~F}_{\mathrm{V}} \mathrm{S}_{1}=\frac{2}{3} \times 2,80 \times 0,30=0,56 \\
& \quad: \mathrm{S}_{\mathrm{DS}}=0,54 \mathrm{~g} \rightarrow \text { table } 6 \\
& ; \mathrm{S}_{D 1}=0,56 \mathrm{~g} \rightarrow \text { table } 7
\end{aligned}
$$

From table 6 and 7 enter $\rightarrow$ Seismic Design CategoryD

Determine the natural vibration time T The fundamental natural vibration time (T) obtained from the results of the analysis of the structural program model is limited not to be greater than the $C_{U} T_{a}$ (SNI 1726-2012) article 7.8.2 and table 14, for Surabaya $\mathrm{S}_{\mathrm{D} 1}=0,56 \rightarrow \mathrm{C}_{\mathrm{U}}=1,4$

$\mathrm{T}_{\mathrm{a}}=\mathrm{C}_{\mathrm{t}} \mathrm{h}_{\mathrm{n}} \rightarrow$ table 15

Direcion - $X, \mathrm{~T}_{\mathrm{a}}=0,0488 \mathrm{x} 26^{0,75}=0,562 \mathrm{sec}$,

So that $T_{X}$ max allowed $=C_{U} T_{a}=1,4 \times 0,562=0,787 \sec ($ direction $X)$

Direction - $\mathrm{Y}, \mathrm{T}_{\mathrm{a}}=0,0466 \mathrm{x} 260,90=0,875 \mathrm{sec}$,

So that $\mathrm{T}_{\mathrm{x}}$ max allowed $=\mathrm{C}_{\mathrm{U}} \mathrm{T}_{\mathrm{a}}=1,4 \times 0,875=1,225 \sec ($ direction $\mathrm{Y}$ )

The natural vibration time is the structure of the results of capital analysis with the SAP program for cross section conditions, according to the provisions ofSNI 03-2847-2002 article 12.11.1

SAP OutputResult Crack condition $\mathrm{T}_{1 \mathrm{X}}=0,874 \mathrm{sec}, \mathrm{T}_{1 \mathrm{Y}}=1,337 \mathrm{sec}$

SAP Output Results Uncrack condition $\mathrm{T}_{1 \mathrm{X}}=0,534 \mathrm{sec}, \mathrm{T}_{1 \mathrm{Y}}=0,844 \mathrm{sec}$

The conditions for calculating the fundamental natural vibration time can be summarized as follows:

\begin{tabular}{|c|c|c|c|}
\hline \multirow{2}{*}{ SNI-03-1726-2012 } & \multicolumn{2}{|c|}{ Analysis from SAP } \\
\cline { 3 - 4 } & & Crack Condition & Uncrack Condition \\
\hline $\mathrm{T}_{1 \mathrm{X}}$ & $0,787 \mathrm{sec}$ & $0,874 \mathrm{sec}$ & $0,534 \mathrm{sec}$ \\
\hline $\mathrm{T}_{1 \mathrm{Y}}$ & $1,225 \mathrm{sec}$ & $1,337 \mathrm{sec}$ & $0,844 \mathrm{sec}$ \\
\hline
\end{tabular}

Table 1

2012.

The maximum vibration time limit is the result of calculations based on the empirical formula ofSNI-03-1726-

$\mathrm{T}_{1 \mathrm{X}}=0,787 \mathrm{sec}, \mathrm{T}_{1 \mathrm{Y}}=1,225 \mathrm{sec}$

Based on SNI-03-1726-2012 article 7.6 and table 13 then,

$\mathrm{T}_{\mathrm{S}}=\mathrm{S}_{\mathrm{D} 1} / \mathrm{S}_{\mathrm{DS}}=0,56 / 0,54=1,037$

$\mathrm{T}_{\mathrm{S}}=1,037 \mathrm{sec} 3,5 \mathrm{~T}_{\mathrm{S}}=3.63$

$\mathrm{T}_{1 \mathrm{X}}=0,787 \mathrm{sec}<3,5 \mathrm{~T}_{\mathrm{S}}$

$\mathrm{T}_{1 \mathrm{y}}=1,225 \mathrm{sec}<3,5 \mathrm{~T}_{\mathrm{S}}$

Building with KDSD, regular, and $\mathrm{T}<3,5 \mathrm{~T}_{\mathrm{S}} \rightarrow$ can use equivalent static analysis procedures

\subsection{Equivalent Static Analysis}

Basic Seismic Load $\rightarrow \mathrm{V}=\mathrm{Cs}_{\mathrm{S}} \mathrm{W}$

$\mathrm{S}_{\mathrm{D} 1}=0,56$

$\mathrm{S}_{\mathrm{Ds}}=0,54 \quad \mathrm{~T}_{1 \mathrm{X}}=0,787 \mathrm{sec}, \mathrm{T}_{1 \mathrm{Y}}=1,225 \mathrm{sec}$

$\mathrm{R}_{\mathrm{X}}=7 \quad$ (table 9)

$\mathrm{R}_{\mathrm{Y}}=8 \quad$ (table 9)

$\mathrm{I}_{\mathrm{E}}=1,5$

\begin{tabular}{|c|c|c|c|c|}
\hline & $\mathbf{C}_{\text {s min }}$ & $\mathbf{C}_{\mathbf{S}}$ & $\mathbf{C}_{\text {S max }}$ & C $_{\mathbf{S}}$ \\
\cline { 2 - 5 } & $0,044 \mathrm{~S}_{\mathrm{DS}} \mathrm{I}_{\mathrm{E}}$ & $\mathrm{S}_{\mathrm{DS}} /\left(\mathrm{R} / \mathrm{I}_{\mathrm{E}}\right)$ & $\mathrm{S}_{\mathrm{DS}} /\left[\mathrm{T}\left(\mathrm{R} / \mathrm{I}_{\mathrm{E}}\right)\right]$ & Used \\
\hline Direction-X & 0,024 & 0,116 & 0,147 & $\mathbf{0 , 1 4 7}$ \\
\hline Direction- $\mathrm{Y}$ & 0,024 & 0,101 & 0,083 & $\mathbf{0 , 0 8 3}$ \\
\hline
\end{tabular}

Table 2

Equivalent Static Earthquake Force Distribution

$\mathrm{F}_{\mathrm{X}}=\mathrm{C}_{\mathrm{VX}} \mathrm{V}$

$C_{V X}=\frac{W x h x^{k}}{\sum_{i}^{n} W i h^{k}}$

$\mathrm{F} \quad$ =Earthquake Load 
$\mathrm{C}_{\mathrm{V}} \quad=$ Distribution coefficient

$\mathrm{h}_{\mathrm{i}} \quad=$ High floor on level i

$\mathrm{W}_{\mathrm{i}} \quad=$ Effective weight on the floor $\mathrm{i}$

$\mathrm{K}$ is for calculating equivalent static

\begin{tabular}{|c|c|}
\hline $\mathrm{T}(\mathrm{sec})$ & $\mathrm{K}$ \\
\hline $\mathrm{T} \leq 0,5$ & 1 \\
\hline $0,5<\mathrm{T}<2,5$ & Interpolation \\
\hline $\mathrm{T} \geq 2,5$ & 2 \\
\hline
\end{tabular}

Table 3

$\mathrm{T}_{1 \mathrm{X}}=0,787 \rightarrow \mathrm{k}=1,07$

$\mathrm{T}_{1 \mathrm{Y}}=1,225 \rightarrow \mathrm{k}=1,36$

Earthquake Scale Factor $=(\mathrm{g}$ x I $) / \mathrm{R}$ :

$\mathrm{R}_{\mathrm{x}}=7 \quad \mathrm{I}=1,5$

$\mathrm{R}_{\mathrm{x}}=8 \quad \mathrm{I}=1,5$

$\mathrm{g}$ (gravity) $=9,81 \mathrm{~m} / \mathrm{sec}^{2}$

Direction $X=100 \%(9,81 \times 1,5) / 7=2,102 \mathrm{~m} / \mathrm{sec}^{2}$

Direction $Y=30 \%(9,81 \times 1,5) / 7=0,630 \mathrm{~m} / \mathrm{sec}^{2}$

Direction $Y=100 \%(9,81 \times 1,5) / 8=1,839 \mathrm{~m} / \mathrm{sec}^{2}$

Direction $X=30 \%(9,81 \times 1,5) / 8=0,552 \mathrm{~m} / \mathrm{sec}^{2}$

3.3. Analysis of Design Spectra Response

Determining Period T

$\mathrm{T}_{\mathrm{S}}=\mathrm{S}_{\mathrm{D} 1} / \mathrm{S}_{\mathrm{DS}}=0,56 / 0,54=1,037$

$\mathrm{T}_{0}=0,2\left(\mathrm{~S}_{\mathrm{D} 1} / \mathrm{S}_{\mathrm{DS}}\right)=0,2(0,56 / 0,54)=0,207$

Determine $\mathrm{Sa}$

For $\mathrm{T}<\mathrm{T}_{0} \rightarrow \mathrm{Sa}=\mathrm{S}_{\mathrm{DS}} \mathrm{X}\left[0,4+0,6 \frac{\mathrm{T}}{\mathrm{To}}\right]$

For $\mathrm{T}<\mathrm{T}_{0}<\mathrm{T}_{\mathrm{S}} \rightarrow \mathrm{Sa}=\mathrm{S}_{\mathrm{DS}}$

For $\mathrm{T}>\mathrm{T}_{\mathrm{S}} \rightarrow \mathrm{Sa}=\frac{\mathrm{SD} 1}{\mathrm{~T}}$

$\mathrm{T}=0 \quad \rightarrow \mathrm{Sa}=0,207$

$\mathrm{T}=\mathrm{T}_{0}=0,207 \rightarrow \mathrm{Sa}=0,540$

$\mathrm{T}=\mathrm{T}_{\mathrm{S}}=1,037 \quad \rightarrow \mathrm{Sa}=0,540$

$\mathrm{T}=1,1 \quad \rightarrow \mathrm{Sa}=0,509$

$\mathrm{T}=1,2 \quad \rightarrow \mathrm{Sa}=0,467$

$\mathrm{T}=1,3 \quad \rightarrow \mathrm{Sa}=0,431$

$\mathrm{T}=1,4 \quad \rightarrow \mathrm{Sa}=0,400$

$\mathrm{T}=1,5 \quad \rightarrow \mathrm{Sa}=0,373$

$\mathrm{T}=1,6 \quad \rightarrow \mathrm{Sa}=0,350$

$\mathrm{T}=1,7 \quad \rightarrow \mathrm{Sa}=0,329$

$\mathrm{T}=1,8 \quad \rightarrow \mathrm{Sa}=0,311$

$\mathrm{T}=1,9 \quad \rightarrow \mathrm{Sa}=0,295$

$\mathrm{T}=2 \quad \rightarrow \mathrm{Sa}=0,280$

$\mathrm{T}=2,1 \quad \rightarrow \mathrm{Sa}=0,267$

$\mathrm{T}=2,2 \rightarrow \mathrm{Sa}=0,255$

$\mathrm{T}=2,3 \quad \rightarrow \mathrm{Sa}=0,243$

$\mathrm{T}=2,4 \quad \rightarrow \mathrm{Sa}=0,233$

$\mathrm{T}=2,5 \quad \rightarrow \mathrm{Sa}=0,224$

$\mathrm{T}=2,6 \quad \rightarrow \mathrm{Sa}=0,215$

$\mathrm{T}=2,7 \quad \rightarrow \mathrm{Sa}=0,207$

$\mathrm{T}=2,8 \quad \rightarrow \mathrm{Sa}=0,200$

$\mathrm{T}=2,9 \quad \rightarrow \mathrm{Sa}=0,193$

$\mathrm{T}=3 \quad \rightarrow \mathrm{Sa}=0,187$

$\mathrm{T}=3,1 \quad \rightarrow \mathrm{Sa}=0,181$

$\mathrm{T}=3,2 \quad \rightarrow \mathrm{Sa}=0,175$

$\mathrm{T}=3,3 \quad \rightarrow \mathrm{Sa}=0,170$

$\mathrm{T}=3,4 \quad \rightarrow \mathrm{Sa}=0,165$

$\mathrm{T}=3,5 \quad \rightarrow \mathrm{Sa}=0,160$

$\mathrm{T}=3,6 \rightarrow \mathrm{Sa}=0,156$

$\mathrm{T}=3,7 \quad \rightarrow \mathrm{Sa}=0,151$

$\mathrm{T}=3,8 \quad \rightarrow \mathrm{Sa}=0,147$

$\mathrm{T}=3,9 \quad \rightarrow \mathrm{Sa}=0,144$

$\mathrm{T}=4 \quad \rightarrow \mathrm{Sa}=0,140$ 
$\mathrm{T}=4.1$

$\rightarrow \mathrm{Sa}=0,137$

Earthquake Scale Factor $=(\mathrm{gx}$ I $) / \mathrm{R}$ :

$\begin{array}{ll}\mathrm{R}_{\mathrm{X}}=7 & \mathrm{I}=1,5 \\ \mathrm{R}_{\mathrm{Y}}=8 & \mathrm{I}=1,5\end{array}$

$\mathrm{g}$ (gravity) $=9,81 \mathrm{~m} / \mathrm{sec}^{2}$

Direction $X=100 \%(9,81 \times 1,5) / 7=2,102 \mathrm{~m} / \mathrm{sec}^{2}$

Direction $Y=30 \%(9,81 \times 1,5) / 7=0,630 \mathrm{~m} / \mathrm{sec}^{2}$

Direction $Y=100 \%(9,81 \times 1,5) / 8=1,839 \mathrm{~m} / \mathrm{sec}^{2}$

Direction $X=30 \%(9,81 \times 1,5) / 8=0,552 \mathrm{~m} / \mathrm{sec}^{2}$

3.4. Building Eccentricity

\begin{tabular}{|c|c|c|c|c|c|c|c|c|c|c|}
\hline \multirow{2}{*}{ Floor } & \multicolumn{2}{|c|}{ Mass Centre } & \multicolumn{2}{|c|}{ Rotation Centre } & \multicolumn{2}{|c|}{ Eccentricity (e) } & \multicolumn{2}{|c|}{ ed $=e+0,05$ Ax (Lx/Ly) } & \multicolumn{2}{|c|}{ ed $=e-0,05 b$ (Lx / Ly) } \\
\hline & $\mathbf{X}$ & $\mathbf{Y}$ & $\mathbf{X}$ & $\mathbf{Y}$ & $\mathbf{X}$ & $\mathbf{Y}$ & $\mathbf{X}$ & $\mathbf{Y}$ & $\mathbf{X}$ & $\mathbf{Y}$ \\
\hline Tie Beam & 12,5 & 43 & 10,68 & 44,45 & 1,82 & 1,45 & 2,91 & 5,22 & 0,73 & $-2,32$ \\
\hline 2nd Floor & 12,5 & 43 & 10,68 & 44,45 & 1,82 & 1,45 & 2,91 & 5,22 & 0,73 & $-2,32$ \\
\hline 3rd Floor & 12,5 & 43 & 10,68 & 44,45 & 1,82 & 1,45 & 2,91 & 5,22 & 0,73 & $-2,32$ \\
\hline 4th F & 12,5 & 43 & 10,68 & 44,45 & 1,82 & 1,45 & 2,91 & 5,22 & 0,73 & $-2,32$ \\
\hline Lantai 5 & 12,5 & 43 & 10,68 & 44,45 & 1,82 & 1,45 & 2,91 & 5,22 & 0,73 & $-2,32$ \\
\hline Lantai 6 & 12,5 & 43 & 10,68 & 44,45 & 1,82 & 1,45 & 2,91 & 5,22 & 0,73 & $-2,32$ \\
\hline Atap & 12,5 & 43 & 10,68 & 44,45 & 1,82 & 1,45 & 2,91 & 5,22 & 0,73 & $-2,32$ \\
\hline Lx & \multicolumn{2}{|c|}{25} & & & & & & & & \\
\hline Ly & \multicolumn{2}{|c|}{86} & & & & & & & & \\
\hline \multicolumn{3}{|c|}{$A x=[\delta \max / 1,2 \delta \text { avg }]^{2}=0,88$} & $<3,0$ & & & & & & & \\
\hline$\delta \max$ & \multicolumn{2}{|c|}{$56,88 \mathrm{~mm}$} & & & & & & & & \\
\hline$\delta \min$ & \multicolumn{2}{|c|}{$44,42 \mathrm{~mm}$} & & & & & & & & \\
\hline
\end{tabular}

Table 4

3.5. Correction of Earthquake Scale Factors

\begin{tabular}{|c|c|c|c|c|c|c|c|}
\hline EQX & LinStatic & & 2633 & 789 & & & \\
\hline EQY & LinStatic & & 691 & 2303 & & & \\
\hline RSPX & LinRespSpe & Max & 1036 & 260 & & & \\
\hline \multirow[t]{3}{*}{ RSPY } & LinRespSpe & Max & 275 & 746 & & & \\
\hline & & \multirow{2}{*}{\multicolumn{2}{|c|}{$\begin{array}{l}85 \% \text { EQX }= \\
85 \% E Q Y=\end{array}$}} & & \multirow{2}{*}{ V Dynamic <85\% V Static } & & \\
\hline & & & & & & & \\
\hline \multicolumn{3}{|c|}{ Multiplier Earthquake Scale Factor Di } & 2,161 & & \multicolumn{2}{|c|}{ Early Spectra Response $=(\mathbf{g} \times I) / \mathbf{R x}=$} & 2,102 \\
\hline \multicolumn{3}{|c|}{ Multiplier Earthquake Scale Factor Di } & 2,624 & & \multicolumn{2}{|c|}{ Early Spectra Response $=(\mathrm{g} \times \mathrm{I}) / \mathrm{Ry}=$} & 1,839 \\
\hline \multirow[t]{3}{*}{ Early } & RPSX U1 & 2,102 & then & 4,542 & & & \\
\hline & RPSX U2 & 0,631 & then & 1,363 & & & \\
\hline & RPSY U1 & 0,552 & then & 1,448 & & & \\
\hline
\end{tabular}

Table 5

3.6. The Calculation Results

Earhquake Slide Force EQX :

\begin{tabular}{|c|c|c|c|c|c|}
\hline Floor & $\begin{array}{c}\text { Effective Weight } \\
\text { (ton })\end{array}$ & $\begin{array}{c}\text { High } \\
(\mathrm{m})\end{array}$ & Wi hik $^{\mathrm{N}}$ & $\begin{array}{c}\text { Fx } \\
(\text { ton })\end{array}$ & $\begin{array}{c}\text { Story Shear } \\
\text { ( ton ) }\end{array}$ \\
\hline Roof & 1357 & 26 & 73431 & 567 & 567 \\
\hline 6 & 1853 & 22 & 85708 & 662 & 1229 \\
\hline 5 & 1853 & 18 & 69146 & 534 & 1763 \\
\hline 4 & 1853 & 14 & 52843 & 408 & 2172 \\
\hline 3 & 1853 & 10 & 36866 & 285 & 2456 \\
\hline 2 & 1853 & 6 & 21343 & 165 & 2621 \\
\hline
\end{tabular}

Table 6 
Earthquake Slide Force EQY

\begin{tabular}{|c|c|c|c|c|c|}
\hline Floor & $\begin{array}{c}\text { Effective Weight } \\
\text { (ton })\end{array}$ & $\begin{array}{c}\text { High } \\
(\mathrm{m})\end{array}$ & Wi hik & $\begin{array}{c}\text { Fy } \\
\text { (ton })\end{array}$ & $\begin{array}{c}\text { Story Shear } \\
\text { (ton })\end{array}$ \\
\hline Roof & 1357 & 26 & 1529933 & 518 & 518 \\
\hline 6 & 1853 & 22 & 1862110 & 631 & 1149 \\
\hline 5 & 1853 & 18 & 1417363 & 480 & 1629 \\
\hline 4 & 1853 & 14 & 1007035 & 341 & 1970 \\
\hline 3 & 1853 & 10 & 637251 & 216 & 2186 \\
\hline 2 & 1853 & 6 & 318124 & 108 & 2293 \\
\hline
\end{tabular}

Table 7

Earthquake Slide Force RSPX :

\begin{tabular}{|c|c|c|c|c|c|}
\hline Floor & $\begin{array}{c}\text { Effective Weight } \\
\text { (ton })\end{array}$ & $\begin{array}{c}\text { High } \\
(\mathrm{m})\end{array}$ & Wi hik & Fx & $\begin{array}{c}\text { Story Shear } \\
\text { ( ton })\end{array}$ \\
\hline Roof & 1357 & 26 & 73431 & 439 & 439 \\
\hline 6 & 1853 & 22 & 85708 & 512 & 951 \\
\hline 5 & 1853 & 18 & 69146 & 413 & 1364 \\
\hline 4 & 1853 & 14 & 52843 & 316 & 1679 \\
\hline 3 & 1853 & 10 & 36866 & 220 & 1899 \\
\hline 2 & 1853 & 6 & 21343 & 127 & 2027 \\
\hline
\end{tabular}

Table 8

Earthquake Slide Force RSPY :

\begin{tabular}{|c|c|c|c|c|c|}
\hline Floor & $\begin{array}{c}\text { Effectivte Weight } \\
(\text { ton })\end{array}$ & $\begin{array}{c}\text { High } \\
(\mathrm{m})\end{array}$ & Wi hik & $\begin{array}{c}\text { Fy } \\
\text { ( ton })\end{array}$ & $\begin{array}{c}\text { Story Shear } \\
\text { ( ton })\end{array}$ \\
\hline Roof & 1357 & 26 & 1529933 & 515 & 515 \\
\hline 6 & 1853 & 22 & 1862110 & 627 & 1143 \\
\hline 5 & 1853 & 18 & 1417363 & 478 & 1620 \\
\hline 4 & 1853 & 14 & 1007035 & 339 & 1960 \\
\hline 3 & 1853 & 10 & 637251 & 215 & 2174 \\
\hline 2 & 1853 & 6 & 318124 & 107 & 2282 \\
\hline
\end{tabular}

Table 9

3.7. Graphic of Slide Force

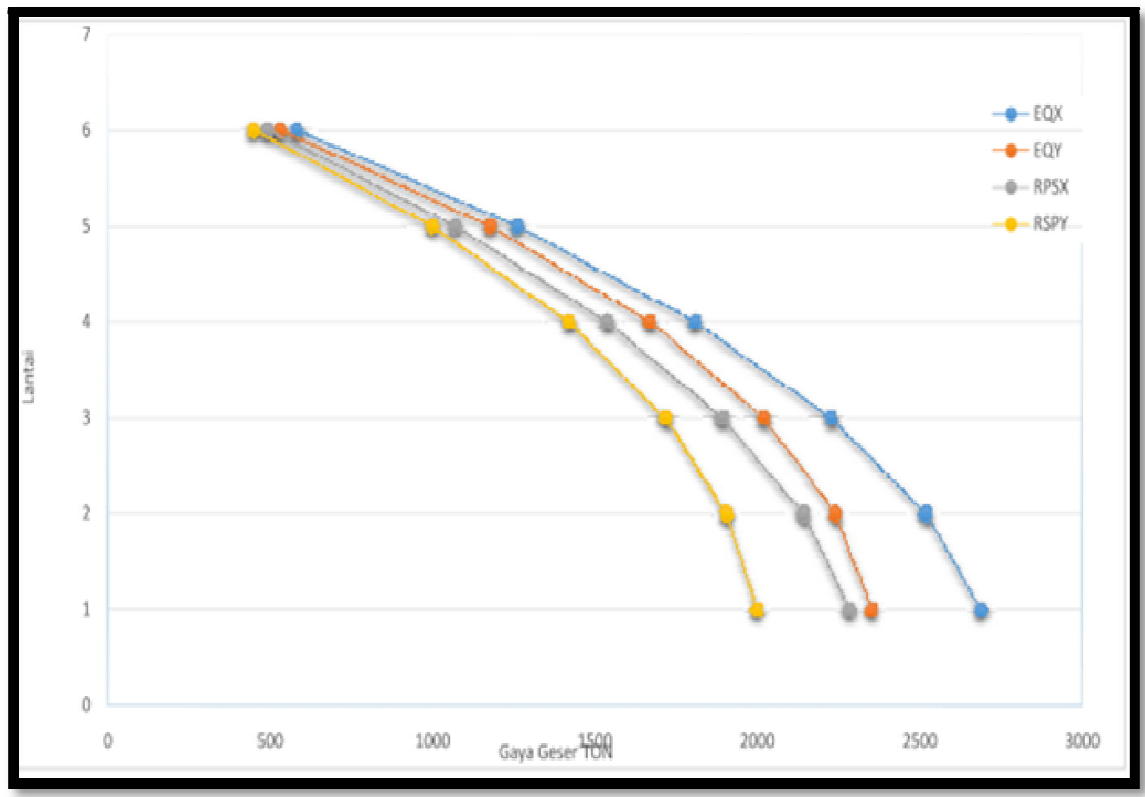

Figure 1 
Deflection at Level X :

$\delta_{\mathrm{X}}=\frac{\mathrm{Cd} \delta \mathrm{xe}}{\mathrm{Ie}}$

$C_{d}=$ deflection enlargement factor, direction $\mathrm{x}=5,5$ direction $\mathrm{y}=5,5$ (in Table 9)

$\delta_{\mathrm{Xe}}=$ deflection determined by elastic analysis

$\mathrm{I}_{\mathrm{e}}=$ priority factor $(1,5)$

\subsubsection{Column Drift}

\begin{tabular}{|c|c|c|c|c|c|c|c|c|}
\hline \multirow{3}{*}{ Floor } & \multirow{3}{*}{$\mathrm{h}(\mathrm{m})$} & \multicolumn{3}{|c|}{ Lateral Load Direction X } & \multicolumn{3}{|c|}{ Lateral Load Direction $\mathrm{X}$} & \multirow[b]{2}{*}{$\Delta \mathrm{a}$} \\
\hline & & $\delta_{x e}$ & $\delta x$ & $\Delta x$ & $\delta y_{e}$ & $\delta y$ & $\Delta y$ & \\
\hline & & $(\mathrm{mm})$ & $(\mathrm{mm})$ & $(\mathrm{mm})$ & $(\mathrm{mm})$ & $(\mathrm{mm})$ & $(\mathrm{mm})$ & $(\mathrm{mm})$ \\
\hline Roof & 26 & 43,33 & 158,88 & 29,92 & 106,66 & 391,09 & 67,61 & 80 \\
\hline 6 & 22 & 35,17 & 128,96 & 31,79 & 88,22 & 323,47 & 76,38 & 80 \\
\hline 5 & 18 & 26,50 & 97,17 & 30,51 & 67,39 & 247,10 & 76,85 & 80 \\
\hline 4 & 14 & 18,18 & 66,66 & 27,43 & 46,43 & 170,24 & 71,43 & 80 \\
\hline 3 & 10 & 10,70 & 39,23 & 22,00 & 26,95 & 98,82 & 56,32 & 80 \\
\hline 2 & 6 & 4,70 & 17,23 & 17,23 & 11,59 & 42,50 & 42,50 & 120 \\
\hline
\end{tabular}

Table 10

3.8 .Determining the Effect of P - $\Delta$

StabilityCoeficient $(\theta$ ) can be calculated based on formula as follows :

$\theta=\frac{\operatorname{Px} \Delta \mathrm{Ie}}{\mathrm{VxhsxCd}}$

Calculation $\theta$ as the effect of $\mathrm{P}-\Delta$

3.8.1. Direction X

\begin{tabular}{|c|c|c|c|c|c|c|}
\hline Floor & $\mathrm{h}(\mathrm{m})$ & $\mathrm{P}$ (ton) & $\Delta \mathrm{x}(\mathrm{mm})$ & $\mathrm{Vx}($ ton) & $\Theta$ & $\theta$ max \\
\hline Roof & 26 & 1357,00 & 29,92 & 437,00 & 0,0006 & 0,25 \\
\hline 6 & 22 & 1853,00 & 31,79 & 948,00 & 0,0005 & 0,25 \\
\hline 5 & 18 & 1853,00 & 30,51 & 1359,00 & 0,0004 & 0,25 \\
\hline 4 & 14 & 1853,00 & 27,43 & 1674,00 & 0,0004 & 0,25 \\
\hline 3 & 10 & 1853,00 & 22,00 & 1894,00 & 0,0004 & 0,25 \\
\hline
\end{tabular}

Table 11

3.8.2. Direction $Y$

\begin{tabular}{|c|c|c|c|c|c|c|}
\hline Floor & $\mathrm{h}(\mathrm{m})$ & $\mathrm{P}$ (ton) & $\Delta \mathrm{y}(\mathrm{mm})$ & $\mathrm{Vy}$ (ton) & $\Theta$ & $\theta \max$ \\
\hline Roof & 26 & 1357,00 & 67,61 & 515,00 & 0,0012 & 0,25 \\
\hline 6 & 22 & 1853,00 & 76,38 & 1143,00 & 0,0010 & 0,25 \\
\hline 5 & 18 & 1853,00 & 76,85 & 1620,00 & 0,0009 & 0,25 \\
\hline 4 & 14 & 1853,00 & 71,43 & 1960,00 & 0,0009 & 0,25 \\
\hline 3 & 10 & 1853,00 & 56,32 & 2174,00 & 0,0009 & 0,25 \\
\hline
\end{tabular}

Table 12

$\Theta<\Theta_{\text {minimum }}=0,10$, Then the Effect of P - $\Delta s$ Ignored

3.9. Soft Strory Examination

\begin{tabular}{|c|c|c|c|c|c|c|c|}
\hline \multirow{2}{*}{ Lantai } & \multirow{2}{*}{$\mathrm{H}_{\mathrm{SX}}(\mathrm{m})$} & \multicolumn{3}{|c|}{ Arah - X } & \multicolumn{3}{c|}{ Arah - Y } \\
\cline { 3 - 8 } & & $\Delta(\mathrm{mm})$ & Drift rasio & $\begin{array}{c}1,3 \text { drif } \\
\text { rasio }\end{array}$ & $\Delta(\mathrm{mm})$ & Drift rasio & $\begin{array}{c}1,3 \text { drif } \\
\text { rasio }\end{array}$ \\
\hline Atap & 4 & 29,92 & 0,007 & 0,010 & 67,61 & 0,017 & 0,022 \\
\hline 6 & 4 & 31,79 & 0,008 & 0,010 & 76,38 & 0,019 & 0,025 \\
\hline 5 & 4 & 30,51 & 0,008 & 0,010 & 76,85 & 0,019 & 0,025 \\
\hline 4 & 4 & 27,43 & 0,007 & 0,009 & 71,43 & 0,018 & 0,023 \\
\hline 3 & 4 & 22,00 & 0,006 & 0,007 & 56,32 & 0,014 & 0,018 \\
\hline 2 & 6 & 17,23 & 0,003 & 0,004 & 42,50 & 0,007 & 0,009 \\
\hline
\end{tabular}

Table 13 
3.10. Dimensions and Reinforcement Required

- Column $60 \times 60 \mathrm{~cm}^{2} 1 \mathrm{st}$ to 6 th floorrequires maximum reinforcement $=8530 \mathrm{~mm}^{2}$ and minimum $=3600 \mathrm{~mm}^{2}$, maximum stirrup $=4,002 \mathrm{~mm}^{2} / \mathrm{mm}$, mimimum $=0,862 \mathrm{~mm}^{2} / \mathrm{mm}$.

- $35 \times 70 \mathrm{~cm}^{2}$ transverse beam, tensile reinforcement is needed $=1798 \mathrm{~mm}^{2}$, compressive reinforcement $=869 \mathrm{~mm}^{2}$, stirrup support $=1,518 \mathrm{~mm}^{2} / \mathrm{mm}$ and pitch crossing $=1,291 \mathrm{~mm}^{2} / \mathrm{mm}$.

- The beams lengthwise $30 \times 50 \mathrm{~cm}^{2}$, tensile reinforcement is needed $=1238 \mathrm{~mm}^{2}$, compressive reinforcement $=858$ $\mathrm{mm}^{2}$, stirrup support $=1,513 \mathrm{~mm}^{2} / \mathrm{mm}$ andpitch crossing $=1,336 \mathrm{~mm}^{2} / \mathrm{mm}$.

- Side shear wall thickness required is $40 \mathrm{~cm}$, vertical reinforcement is $2 \mathrm{D} 16$, distance is $150 \mathrm{~mm}$, Horizontal reinforcement $2 \mathrm{D} 13$, distanceis $150 \mathrm{~mm}$.

- Shear wall lift thickness required is $30 \mathrm{~cm}$, vertical reinforcement is $2 \mathrm{D} \mathrm{16}$, distance is $150 \mathrm{~mm}$, horizontal reinforcement is $2 \mathrm{D} 13$, distance is $150 \mathrm{~mm}$

- The number of foundations on the Shear Wall section needs to be added.

\section{Conclusion}

From the evaluation results can be concluded:

- The 60/ 60 column dimension still meets the requirements, for reinforcement and stirrups some need to be added.

- 35/ 70 transverse beams both dimensions and longitudinal bars and stirrups still meet the requirements.

- The bars extend 30/50, longitudinal bars and stirrups need to be added.

- Shear Wall, both thickness and reinforcement need to be added.

- Shear Wall foundation needs to be added.

\section{References}

i. Earthquake Resistant Planning Regulation SNI 031726 - 2012

ii. Planning Reinforced Concrete SNI 032847 - 2013

iii. Jack C. McCormac, the 5th Edition Reinforced Concrete Design,

iv. Rachmat Purwono, Planning for Earthquake Resistant Reinforced Concrete Structures.

v. 5.Planning Documents for Menur Surabaya Mental Hospital 2017

vi. 2017 Indonesian Earthquake Source and Hazard Map 